\title{
Research on Achieving Data Security with the Cloud Computing Adoption Framework
}

\author{
R.Adhi Lakshmi, P.Priyadharshini, R.Bhaskaran
}

\begin{abstract}
- offering perpetual statistics safety for petabytes of facts is important for handed on enrolling. A ordinary overview on cloud safety imparts that the safety of customers' records has the maximum lifted need and moreover challenge. We acquire this need in order to accomplish with a manner of thinking this is succesful, adoptable and all around made. consequently, this paper has constructed up a device known as Cloud Computing Adoption Framework (CCAF)which has been modified for verifying cloud information. This paper clears up the chart, device for deduction and regions within the CCAF to make certain statistics safety. CCAF is outlined through using the framework route of action depending on the fundamentals and the execution confirmed up thru the CCAF multi-layered protection. while you do not forget that our records center has 10 petabytes of data, there can be splendid challenge to provide nonstop safety and detach. We use business agency manner Modeling Notation (BPMN) to mirror how information is getting used. using BPMN redirection allows us to assess the picked protection introductions in advance than showed execution. outcomes show that an opportunity to assume duty for security break may have a few spot inside the extent of 50 and 100 twenty 5 hours. The server farms have skilled problems of brisk increase within the data. as an example, in a server broaden that the lead writer used to art work with, properly ordered option of 100 terabytes of statistics modified into elegant this proposes greater safety is needed to guarantee all statistics is particularly ensured inside the squeezing a hundred twenty five hours. This paper has correspondingly tested that $C C A F$ multi-layered protection can ensure statistics coherently and it has 3 layers of safety: 1) firewall and get admission to govern; 2) personality affiliation and obstruction adjusting movement and 3) joined encryption. To avow CCAF, this paper has gotten a deal with on techniques of proper hacking assessments required with attack trying out with 10,000 Trojans and pollutions. The CCAF multi-layered security can rectangular 9,919 illnesses and Trojans which can be beaten like a burst and the staying ones may be disengaged or disconnected. The tests seem paying little admire to the way in which that the diploma of blockading can decrease for steady aggregate of ailments and Trojans, 90 seven.forty three percent of them can be restricted. Our CCAF multi-layered safety has a humdrum of 20 percentage ideal execution over the singlelayered manner of thinking which can simply block7, 438 defilements and Trojans.
\end{abstract}

key terms - CCAF Cloud Computing Adoption Framework, BPMN agency technique Modeling Notation, ABE characteristic based Encryption

Revised Manuscript Received on April 12, 2019.

R.Adhi Lakshmi, Assistant Professor, Department of Information Technology, PSNA College Of Engineering \& Technology Dindigul, T.N, India. (adhikarthickpsna@psnacet.edu.in)

P.Priyadharshini, Assistant Professor, Assistant Professor, Department of Information Technology, PSNA College Of Engineering \& Technology Dindigul, T.N, India. (priya@psnacet.edu.in)

R.Bhaskaran, Associate Professor, Assistant Professor, Department of Information Technology, PSNA College Of Engineering \& Technology Dindigul, T.N, India. (rbhaskaran @ psnacet.edu.in)

\section{CREATION}

In conventional structures control plans, if a device substance needs to get to some information content, it desires to discover and companion with the server that gives such management following gadget guidance conventions. ultimately, the information is firmly related with the location of the server. The complete system is targeted at the institutions among substance customers and substance providers, making affiliation repute a massive component to the machine. In ICN engineering, the center is moved from purchaser server establishments with consumer content material cloth institutions. in this way, in-stead of spotting the substance proprietor's location, the system adjustments to distinguish bona fide substance duplicates. Thusly, the purchasers don't have to apprehend wherein the substance locates,i.e. the IP cope with of the substance proprietor. The substance call is ok to guide the client to a substance duplicate. content material owners distribute the substance, which may be replicated and placed away in the device making use of system stores. This shape empowers substance being proficiently conveyed to purchasers. The proposed plan may be isolated into degrees. at the topstage, to deal with the trait the board trouble, we gift a philosophy primarily based absolutely ascribe the board answer for cope with the conveyed houses in ICN installation. on this plan, houses characterised via numerous specialists may be synchronized more correctly than common methodologies. content material customers do not should arrange their trait keys after they solicitation substance from one-of-a-kind professionals. at the decreasestage, we recommend an ABE-primarily based naming plan. 1.1 Attribute Based Encryption (ABE) schemes

In our method, each tool detail is alloted with hundreds of houses with the assistance of a relied on zero.Trusted Third Party (TTP) accord-in to their genuine characters. the entrance manipulate approach is carried out via the substance names instead of the substance. further, protection safeguarding is accommodated the substance get to techniques. This detail can significantly beautify the safety guarantee on ICN records whilst they're conveyed within the open region. along the ones lines, a consumer can distinguish its qualification of the have been given to substance via the scrambled names earlier than virtually attending to the data content material cloth. To further help 
the usage of philosophy in trait the executives, the proposed plan empowers. examination among trends, which gives the potential to rank traits and accomplice numerous blessings as desires be. In rundown, the commitments of our work can be recorded as pursues: It offers philosophy based fine administration, which considerably decreases the fee for trait the board in dispersed business enterprise. The proposed administration plan underpins adaptable belongings blend sports in get right of entry to control arrangements; it empowers satisfactory rankings and get admission to gain the board, making it adaptable to assemble an statistics get to method in real scenario. The substance get to approach is privately protected. Ineligible clients cannot decide the information get to arrangements irrespective of whether or not or now not they plot collectively. It proposes a naming plan for ICN set up which consolidates the adaptable trait the board affiliation with the protection saving get entry to technique. It essentially diminishes the calculation and correspondence overhead for a ability patron to decide his qualification to get to the substance. maximum of those ICN arrangements middle across the effectiveness and safety additives of the tool whilst get admission to govern to the substance and substance safety isn't always all spherical tended to. In, an through a phase known as the Relaying party (RP). a similarly segment called get admission to govern corporation (ACP) is liable for making get right of access to techniques and authorizing the processes to consumers' certifications. This framework joins get proper of entry to control into ICN frameworks however requires substantially extra machine among sports activities. For substance safety functions, proposes a plan in which every report is isolated into squares.

\section{ASSOCIATED PAINTINGS}

Dispersed figuring and its accumulating has been a subject of exchange inside the beyond couple of years. it has been an concept for revolutionary amassing in mild of options in fee-reserves, development in artwork efficiencies, enterprise deftness and nature of groups.With the snappy climb in circulated figuring, programming as an business enterprise (SaaS) is mainly broadly identified, because it offers advantages that match customers' need. as an instance, fitness informatics can empower recovery investigators to break down trying out ailments and tumors. coins related examination can make sure precise and rapid propagations to be open for budgetary authorities. guidance as an business enterprise improves the concept of getting ready and transport. flexible applications empower customers to play 8db290b6e1544acaffefb5f58daa9d83 beguilements and easy to-use programs to amongst act with their allies. while extra humans and affiliations use the cloud groups, security and guarantee turn out to be essential to make certain that all of the statistics they use and provide are all around assured. a couple of masters explicit that safety have to be finished before the usage of any cloud advantages in vicinity. This makes a finding out appointment situation for courting considering safety must be standard and accomplished in parallel with any agencies. no matter the manner that affiliations that get circulated figuring recognize blessings furnished through cloud organizations, stressful conditions, for instance, safety and coverage remain an examination for definitive allocation. whilst coordinating the centrality of safety, the object building and development technique have to continuously association, execute and test protection capabilities. The server homesteads have encountered demanding situations of snappy augmentation within the statistics. for example, in a server ranch that the lead maker used to paintings with, constantly growth of 100 terabytes of statistics changed into commonplace.If the affiliation has encountered a short rising of statistics improvement and cannot respond quick and capably, issues, as an example, records development, records safety and organization degree assention troubles can occur. in this paper, we base on the statistics security at the same time as experiencing a generous augmentation of facts, paying little respect to whether or no longer they may be from the external resources, as an example, attack of sicknesses or Trojans; or they from the inward belongings if clients or customers carry collectively severa terabytes of data continuously. this is ask approximately take a look at for data safety that's high for the higher corporation of the server residing residence to manage a quick augmentation inside the records. The future growth to art work with the going with :

i)to deal with with hacking of facts

ii)Non-lifestyles

iii) records management in keeping with storing

iv) Scalability.

\section{CCAF CHOICE}

on this method, record can be encoded with the help of "DES" computation. subsequent approach, we are capable of store the scramble archive into CSP.CSP is one of the server this is used for the restriction approach. This little little bit of leeway has introduced to its recognition in cryptographic programming. virtual system can offer a path set plan that fluctuates from veritable laptop's; smooth help, software program provisioning, availability and accommodating healing. in the end, the document can be in genuine manner. systems and software program software protection making plans oversees constructing security in CCAF best from necessities. that is moreover visible as making programming packages with CCAF. 


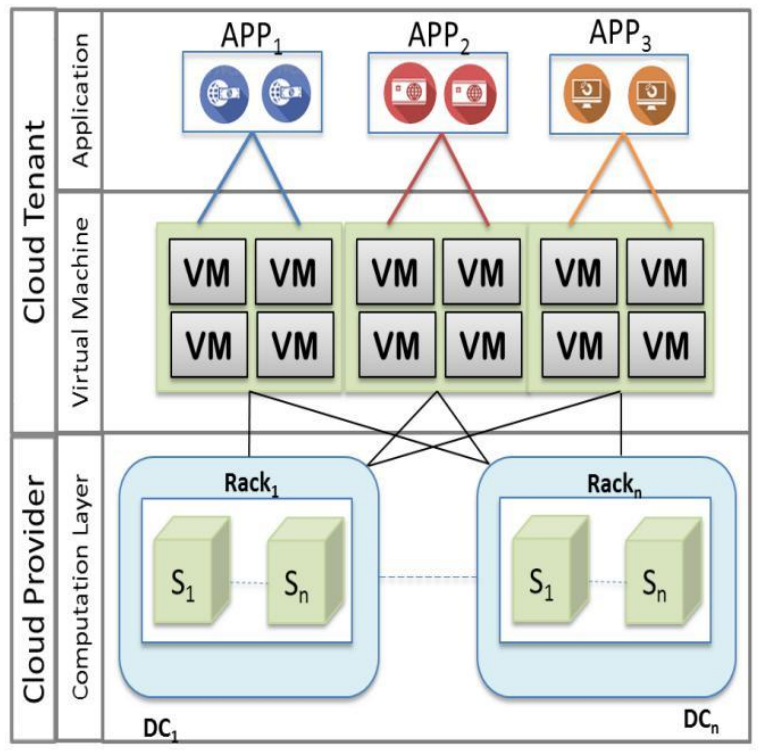

Figure 1.System Architecture

\subsection{Application Layer}

Software program layer security insinuates methodologies for making sure web applications on the software (layer 7 of the seven-layer OSI display) from toxic assaults that might reveal private facts. safety is related to the software layer mainly to assure against unapproved access and assaults. software degree safety indicates the ones protection benefits which is probably invoked on the interface among an software and a line govt to which it's miles related. software program stage protection is usually called begin to finish protection or message stage safety.

\section{2 digital system}

A digital device (VM) is a running framework (OS) or software program condition this is introduced on programming, which copies dedicated device. The give up client has a similar come across on a virtual device as they would have on committed gadget. most digital machines are facilitated on, or works within, a physical gadget. For digital gadget to paintings, the facilitated physical system offers programming referred to as hypervisor. The digital device capacity is a functionality for the acknowledgment of digital device condition. This ability empowers you to make numerous loose virtual machines on one bodily tool by using the usage of virtualizing belongings, as an example, the CPU, reminiscence, device and circle which can be brought on a bodily machine.three.three Computation Layer

Arranging is an important problem in disseminated processing. To run faraway desktops, booking is a essential errand. Many amount of complex applications are arriving strain inside the path of disseminated registering. Conveyed figuring is a essential perspective to revel in some distance flung center interests. reserving troubles are on an essential degree of cloud arranging. This explores the various reserving problems in cloud arranging and specific cuttingedge fashions to apprehend them. on this, first-rate computational model of conveyed processing, arranging troubles are

\section{MODULE DESCRIPTION \& RESULTS}

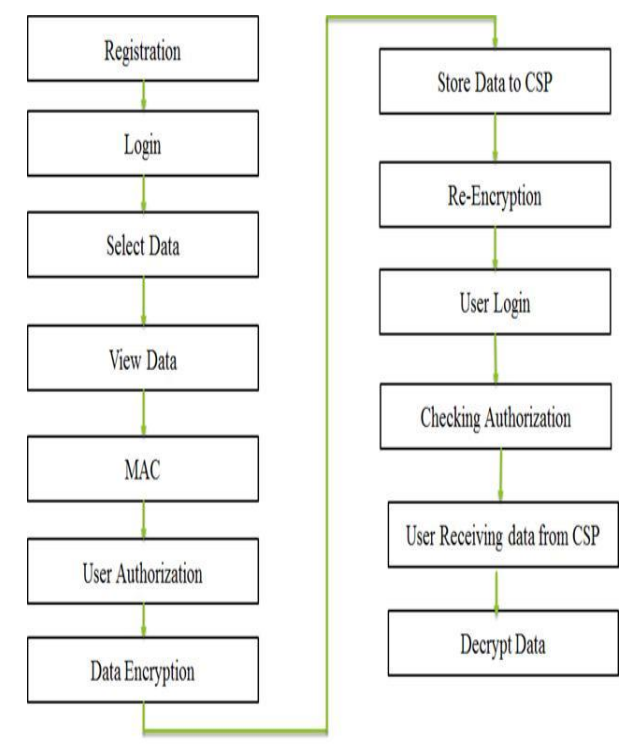

\subsection{INITIAL PHASE:}

Within the underlying degree, the login technique are finished. in the event that the consumer is new consumer implies, the enrollment way perfumed and the customer will enlist their records into the database. again the purchaser login device are completed. After that the report may be positioned away efficiently.

\section{four. 2 content owner'S records:}

Content material fabric proprietor want to pick out the statistics and the statistics might be at any configuration. at the off danger that the facts is in pdf or ppt doc means that records are changed over into content association. At that component it will probably be encoded through a similar way.

\section{4.three content material owner ENCRYPTION:}

Information owner creates the message validation code for the records.This MAC is utilized as the key for the encryption of the information.For encryption information Encryption cutting-edge calculation (DES) is used.It makes use of 56 bits as key size and furthermore the information owner ship the approval guidelines. It includes the name of the beneficiaries. just the ones legal customers can get the records from CSP.

\section{four.4 CSP RE-ENCRYPTION:}

CSP (Cloud provider provider) gets the accompanying substance from facts owner. they may be

\section{- $\quad$ Encrypted facts. \\ - $\quad$ Authorization pointers. \\ - Re-Encryption keys.}

Using the re encryption key the scrambled statistics have be encoded again.Then the cloud specialist co-op is ready to offer management to the records consumer.Cloud benefit supplier gets the demand from statistics consumer.This re encryption gadget builds the security of the facts. 


\section{four. 5 consumer Retrieval:}

Statistics consumer must login into the device.And send the request to the cloud carrier provider.Cloud company company validates the request from the records person. For this validation system the authorization policies are used. If the facts customer call is gift within the authorization recommendations method the CSP will offer the information. in any other case CSP will no longer provide the statistics. Then the facts user decrypts the data received from the cloud issuer employer.

\section{CONCLUSION}

This paper installed the CCAF multi-layered safety for the information safety in the statistics middle underneath the pro-postal and advice of CCAF suggestions. We explained the motive, evaluate, additives in the CCAF, in which the format became based totally at the necessities and the implementation changed into illustrated by using way of its multi-layered protection. We defined how multi-layered safety changed into a suitable approach and recommendation, because it offered multiple pro-segment and improvement of protection for $10 \mathrm{~PB}$ of records in the statistics middle based totally on the university of London Computing middle. We explained the technical facts in every layer of protection and advise an included answer to check all of the information even as records is intensively used. We used the business enterprise gadget Modeling Notation to simulate the times of the manner the data may be used, both at relaxation, in use, or in motion. All simulations may be finished within 2 seconds. Our BPMN simulation outcomes showed that it can soak up to 50 hours to defend all of the 2 PB information and up to125 hours to elevate an alarm to take manage of the situation in the ULCC information middle. because of this an blanketed approach became required to ensure facts protection, in case that the facts center is under the attack or ability danger from the rapid rise of data growth inside the facts center, which comparison between CCAF and different singleLayered offerings Precision don't forget F-degree can be because of the outside intrusion or the inner rapid intake. We then used FGSM for the penetration test-in. 10,000 viruses and Trojans have been injected into information Cen-term with experiments accomplished. the primary experiment showed that firewall, identification manage and encryption may want to block five, 423 , three, 742 and 842 viruses and Trojans respectively. The remaining eighty one can be both quarantined or isolated. the second one test showed that continuous injection of 10,000 viruses and Trojans need to make the block-in price decreased from the 99.19 to 76.00 percent in one hundred twenty five irrespective of of this result, the CCAF multi-layered protection should quarantine and isolate 97 .fifty 3 percent of viruses and Trojans. Our paintings can display that the usage of CCAF multi-layered safety can shield the data center from the rapid information boom because of the protection breach, and the use of BPMN can calculate how lots time required for rescue motion if the information safety is compromised. in this manner, we will training session the better tactics and plans for statistics recovery and safety.

\section{REFERENCES}

1. S. Marston, Z. Li, S. Bandyopadhyay, J. Zhang, and A Thalassic, "Cloud computing - The enterprise mindset," selection assist Syst., vol. 51, no. 1, pp. 176-189, 2011.

2. M. A. Vouch, "Cloud computing-issues, research and implementations," J. Compute. Inf. Technol. - CIT, v o 1. four, p p. $235-246,2$ zero 08.

3. A. adequate. Johan, C. M. DesRoches, E. G. Campbell, okay. Don élan, S. R. Rao, T. G. Ferris, and D. Blumenthal, "Use of electronic fitness data In US hospitals," New England J. Med., vol. 360, no. sixteen, pp. 1628-1638, 2009.

4. H. T. Peng, W. W. Hsu, C. H. Chen, F. Lai, and J. M. Ho, "monetary Cloud: open cloud framework of by-product pricing," in Proc. Int.Conf. Social Compute. Sep. 2013, pp. 782-789.

5. M. Marcia and A. I. Andreessen, "the use of cloud computing in better training: A method to improve agility in the cuttingedge financial crisis, "commonplace. IBIMA, vol. 2011, pp. $1-15,2011$.

6. M. Armrest, A. Fox, R. Griffith, A. D. Joseph, R. H. Katz, Kaminski, G. Lee, D. Patterson, A. Rabin, I. Stoical, and M. Zaharias, "Above the clouds: A Berkeley view of cloud Computing," not unusual. ACM, vol. fifty three, no. four, pp. 50- fifty 8, 2010.

7. L. Liu, E. Yu, and J. Mylopoulos, "safety and privacy requiremints evaluation inner a social setting," in Proc. 11th IEEE Int. requirements Eng. Conf., Sep. 2003, pp. 151-161.

8. T. Mather, S. Kumaraswamy, and S. Latin, Cloud protection and privateness: An organization attitude on dangers and Compliance. Sebastopol, CA, u.s.: O’Reilly Media, 2009.

9. M. Pop and S. L. Salzburg, "Bioinformatics traumatic situations of latest Sequencing generation, "TrendsGenetics, vol. 24, no. three, pp.

10. Q. Zhang, L. Cheng, and R. Boothbay, "Cloud computing: modern-day and studies demanding situations," J. internet offerings Appl., vol. 1,No. 1, pp. 7-18, 2010.

11. J. J. Cibola and L. R. younger, "A taxonomy of operational cyber protection," tender. Eng. Inst., Tech. note: CMU/SEI2010-TN-028, Pittsburgh, PA, u.s., Dec. 2010.

12. S. Yu, C. Wang, good enough. Ren, and W. Lou, "conducting comfy, scalable, And exceptional-grained information get proper of access to control in cloud computing," in Proc. IEEE INFOCOM, Mar. 14-19, 2010, pp. 1-9.

13. G. Wang, Q. Liu, and J. Wu, "Hierarchical characteristicprimarily based completely encryption for fantastic-grained get admission to govern in cloud garage offerings," in Proc. seventeenth ACM Conf. Compute. commonplace. protection, Oct. 2010, pp. 735-737.

14. G. McGraw, software program protection: building protection. reading, MA, u.s.: Addison-Wesley, 2006.

15. P. Brooks and J. Chittenden, Metrics for provider manage: Designing for ITIL. Zaltbommel, Netherlands: Van Harem submit-in, 2012.

16. V. Chang, R. J. Walters, and G. Wills, Cloud storage and Bioinformatics in a personal Cloud Deployment: training for facts in depth research. big apple, big apple, u.s.: Springer closer 2012, CCIS 367, pp. 245-264, 2013.

17. V. Chang, "business company intelligence as company inside the cloud," future genre. Compute. Syst., vol. 37, pp. 512534,2014

18. I. A. Toned et al., "safety necessities for relaxation humans: A survey," IEEE soft., specific difficulty safety Agile Requirement Eng. methods, vol. 25, no. 1, pp. 20-27, Jan. /Feb. 2008.

19. N. R. Mead, T. Stoney, "safety exceptional requirements engineering (square) technique," AC M, vol. 30, no 4, pp. 1$7,2005$. 
20. Oracle. (2012). statistics safety demanding situations. Oracle9i safety over-view launch range 2(nine.2), accessed on 4th November nline].to be had:

21. V. Kumar, M. S. Swathe, M. S. Muneshwara, and S. Prakash "Cloud computing: closer to case study of facts safety mechanism," Int. J. Adv. Technol. Eng. Res., vol. 2, no. four, pp. 1-eight, Jul. 2012.

22. F. Wen, and L. Xiang, "The have a look at on data protection in cloud com-putting based totally totally on virtualization," in Proc. IEEE Int. Sump. IT Med. Edu. , 2011, vol. 2, no. 1, pp. 257-261.

23. B. Schneider, beyond worry. huge apple, massive apple, u.s.: Copernicus Books,2003.

24. IBM, "11 conduct for specially a success BPMprograms," IBM-notion control White Paper, 2010.

25. G. M. Camino and G. Tagline, "An interval-valued approach to business organisation procedure simulation primarily based on genetic algorithms and the BPMN," data, vol. five, pp. 319-356, 2014.

26. A. Buhl and k. Buhl, "An assessment of cloud computing safety issues," in Proc. worldwide Conger. Inf. not unusual. Technol., Trivandrum, India, Nov. 2012, pp. 109-114.

27. V. Vardharajan and U. Tupakula, "protection as a company model for cloud environment," IEEE Trans. Newt. carrier manage., vol. 11,No. 1, pp. 60-75, Mar. 2014.

28. M. Bishop, "approximately penetration finding out," IEEE protection privacy ,vol. five, no. 6 , pp. eighty four-87, Nov. /Dec. 2007. 